\title{
Monitoração terapêutica da azatioprina: uma revisão
}

\author{
Therapeutic drug monitoring of azathioprine: a review
}

Maurílio Pacheco Neto'; Atecla Nunciata Lopes Alves²; Alexandre Soriano Fortini33; Marcelo do Nascimento Burattini4; Nairo Massakazu Sumita5; Miguel Srougi ${ }^{5}$; Pedro Renato Chocair ${ }^{7}$

\begin{abstract}
unitermos
\section{resumo}

Azatioprina

6-mercaptopurina

6-tioguanina

Imunossupressão

Cromatografia líquida

Monitorização de

medicamentos

Os nucleotídeos de tioguanina (6-TGN), metabólitos ativos da azatioprina (AZA) e da 6-mercaptopurina (6-MP), atuam como antagonistas das purinas, inibindo as sínteses de DNA, RNA e a protéica, e induzindo à citotoxicidade/imunossupressão. A enzima geneticamente determinada, tiopurina metiltransferase (TPMT), está envolvida no metabolismo desses agentes e, hipoteticamente, determina a resposta clínica às tiopurinas. A baixa atividade dessa enzima diminui a metilação das tiopurinas, resultando em potencial sobredose, enquanto altos níveis de TPMT levam à superprodução do metabólito tóxico 6-metilmercaptopurina (6-MMP) e à não-efetividade terapêutica da AZA e da 6-MP. Várias mutações no gene da TPMT têm sido identificadas e correlacionadas com fenótipos de baixa atividade. Neste artigo, também se discute a monitoração terapêutica desses fármacos por meio da medida dos níveis de 6-TGN intra-eritrocitários, os quais se correlacionam com imunossupressão e mielotoxicidade. Já a 6-MMP está diretamente relacionada com hepatotoxicidade. Esses ensaios estão associados ao uso de doses adequadas dessa droga, resultando num melhor controle da doença e menor uso de corticosteróides.
\end{abstract}

abstract

Thioguanine nucleotides (6-TGN), active metabolites of azathioprine (AZA) and 6-mercaptopurine (6-MP), act as purine antagonists, inhibiting DNA, RNA, and protein synthesis and inducing cytotoxicity and immunosuppression. The genetically determined thiopurine methyltransferase enzyme (TPMT) is involved in the metabolism of these agents and, theoretically, determines the clinical response to thiopurines. Low activity of this enzyme decreases the methylation of thiopurines, what results in potential overdosing, whereas high TPMT status leads to overproduction of toxic metabolite 6-methilmercaptopurine (6-MMP) and ineffectiveness of AZA and 6-MP. Several mutations in the TPMT gene have been identified and correlated with low activity phenotypes. In this study, we also discuss the therapeutic monitoring of these drugs by means of red blood cell 6-TGN levels, which correlate with immunosuppression and mielotoxicity. 6-MMP is directly connected with hepatotoxicity. These metabolites assays are associated with the use of appropriate doses of this drug, what results in a better control of the disease and a decreased use of corticosteroids. key words

6-mercaptopurine

6-thioguanine

Azathioprine

Drug monitoring

Immunosuppression

Liquid chromatography

\footnotetext{
1. Mestrando em Fisiopatologia Experimental pela Faculdade de Medicina da Universidade de São Paulo (FMUSP); pós-graduando do Setor de Toxicologia do Serviço de Bioquímica Clínica da Divisão de Laboratório Central do Hospital das Clínicas da FMUSP (DLC/HC/FMUSP). LIM-03 da Patologia Clínica da FMUSP.

2. Doutora em Toxicologia e Análises Toxicológicas pela Faculdade de Ciências Farmacêuticas da Universidade de São Paulo (FCFUSP); biologista do Setor de Toxicologia do Serviço de Bioquímica Clínica da DLC do HC/FMUSP. LIM-03 da Patologia Clínica da FMUSP.

3. Pós-graduado em Bioquímica Clínica e Imunologia; médico assistente da DLC do HC/FMUSP. Prédio dos Ambulatórios. Av. Dr. Enéas de Carvalho Aguiar, 255. $2^{\circ}$ andar.

4. Professor livre-docente do Departamento de Patologia da FMUSP; diretor da DLC do HC/FMUSP. LIM-03 da Patologia Clínica da FMUSP.

5. Doutor em Patologia pela FMUSP; diretor do Serviço de Bioquímica Clínica da DLC do HC/FMUSP. LIM-03 da Patologia Clínica da FMUSP; professor colaborador da Disciplina de Patologia Clínica da FMUSP.

6. Professor titular da Disciplina de Urologia da FMUSP.

7. Professor livre-docente em Nefrologia pela FMUSP; médico assistente da Clínica de Urologia - Unidade de Transplante Renal (UTR) - do HC/FMUSP.

Trabalho realizado no Setor de Toxicologia do Serviço de Bioquímica Clínica da DLC/HC/FMUSP.
} 


\section{Introdução}

Este artigo de revisão tem por objetivo descrever a importância da monitoração terapêutica da azatioprina (AZA), fundamentada nos estudos farmacocinéticos e nas metodologias desenvolvidas para esse fim.

A AZA foi desenvolvida na década de 1950 e, desde então, é alvo de inúmeros estudos. Sua principal ação farmacológica deve-se à incorporação do metabólito ativo - a 6-tioguanina (6-TGN) - ao DNA das células. A 6-TGN possui estrutura análoga às bases púricas, adenina e hipoxanti$\mathrm{na}^{(21,24)}$. É utilizada na terapia medicamentosa de pacientes com doenças inflamatórias intestinais (DIIs), leucemia linfóide aguda (LLA) e na imunossupressão pós-transplante. Em razão do seu efeito antiinflamatório, também tem sido utilizada em pacientes com doenças auto-imunes, como lúpus, vasculites, alterações dermatológicas e artrite reumatóide (AR) ${ }^{(2632)}$.

Alguns aspectos da sua função terapêutica permanecem obscuros por conta do seu complexo metabolismo e do polimorfismo genético da enzima tiopurina metiltransferase (TPMT). Os pacientes submetidos à terapia com AZA apresentam amplas variações nos níveis de 6-TGN intra-eritrocitários, mesmo quando submetidos a doses semelhantes. Entre 9\% e 25\% dos pacientes em tratamento de Dlls abandonam a terapia devido aos efeitos tóxicos, apontando assim a importância do estudo toxicocinético desse imunossupressor ${ }^{(20,26)}$.

Nos países desenvolvidos, a incidência de Dlls, como a doença de Crohn (DC), aproxima-se de 50 casos para cada 100 mil habitantes. No Brasil, como em outros países emergentes, a incidência também vem aumentando ${ }^{(8)}$.

Em relação à imunossupressão pós-transplante induzida pela AZA, é importante reconhecer que o conhecimento atual necessita ser aprimorado por meio de estudos da atividade enzimática, da genotipagem da TPMT e da monitoração terapêutica de seus metabólitos ativos e tóxicos. Tal necessidade se torna ainda mais evidente quando se leva em conta que o Brasil possui o maior sistema público de transplantes do mundo e que em 2006 foram realizados mais de 10 mil transplantes ${ }^{(35)}$. Além disso, todos os anos surgem novos medicamentos imunossupressores, com as mais variadas promessas de avanços terapêuticos. $\mathrm{O}$ protocolo de tratamento depende de constantes estudos e cotejo entre os resultados.

\section{Farmacocinética e farmacodinâmica}

Na maioria dos casos administram-se doses orais de 2 ou $2,5 \mathrm{mg} / \mathrm{kg} / \mathrm{dia}$ na forma de comprimidos de $50 \mathrm{mg}$, com doses iniciais de AZA entre 50 e $200 \mathrm{mg}$ diárias ${ }^{(25,33)}$. Tanto a AZA quanto a 6-mercaptopurina (6-MP) apresentam absorção gastrintestinal, baixa biodisponibilidade e meia-vida plasmática muito curta (entre uma e duas horas). Cerca de $30 \%$ do medicamento circula ligado às proteínas plasmáticas, entretanto a meia-vida dos nucleotídeos da 6-TGN intra-eritrocitários varia entre três e 13 dias. Esse fato pode explicar a demora de cerca de três ou quatro meses para que níveis estáveis de 6-TGN sejam alcançados no tratamento com AZA.

Com distribuição ampla por todos os líquidos corporais, em média $88 \%$ da AZA são convertidos em 6-MP(2, 34) pela ação da glutationa, um sistema hepático enzimático catalisado pelas enzimas glutationa transferases (GSTs) ${ }^{(18)}$. Após captação celular, o metabolismo da 6-MP segue por três vias principais, formando os seguintes compostos (Figura 1):

- ácido tioúrico (6-TU) pela ação da enzima xantina oxidase (XO) no fígado e na mucosa intestinal;

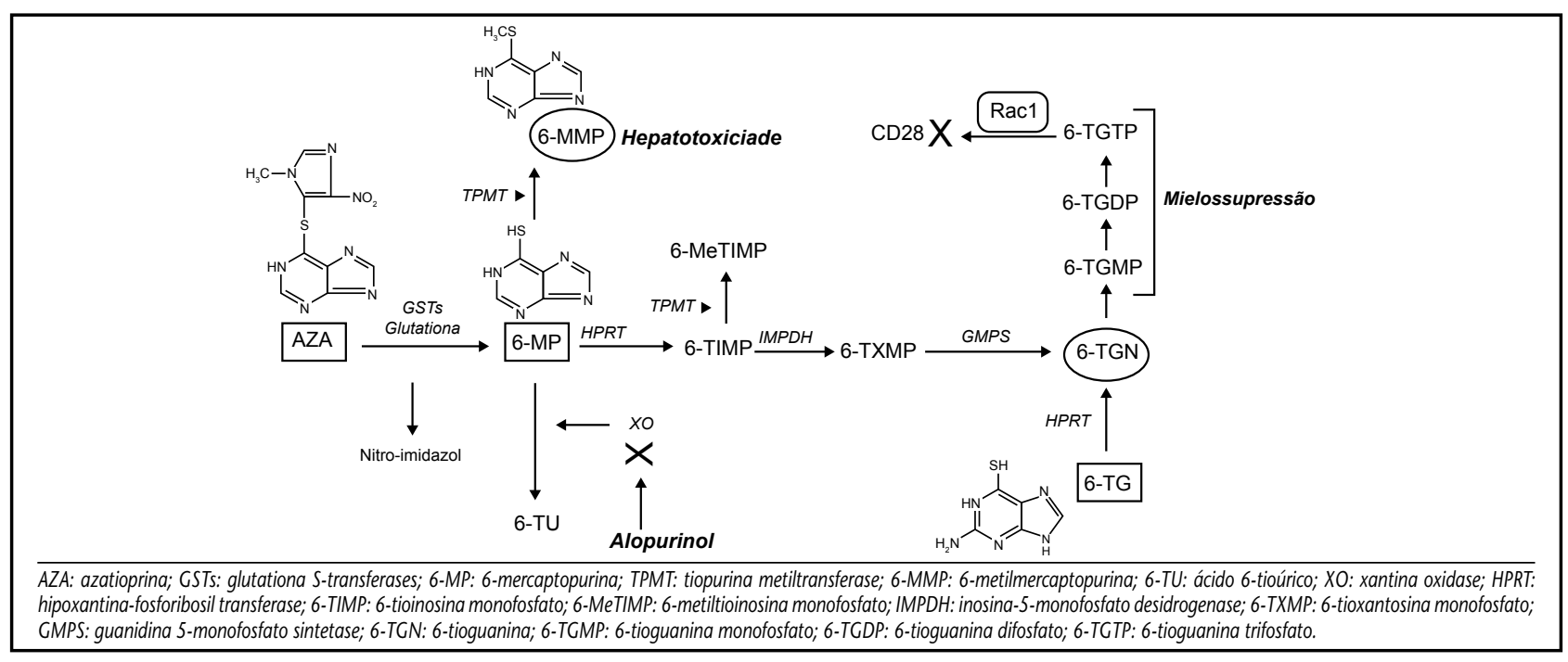

Figura 1 - Representação esquemática do metabolismo da azatioprina 
- 6-metilmercaptopurina (6-MMP) pela ação da TPMT intra-eritrocitária;

-6-TGN e seus nucleotídeos ativos: 6-tioguanina monofosfato (6-TGMP), 6-tioguanina difosfato (6-TGDP) e 6-tioguanina trifosfato (6-TGTP).

Nessa etapa, a 6-MP é transformada pela hipoxantinafosforibosil transferase (HPRT) em 6-tioinosina monofosfato (6-TIMP), que pode seguir dois caminhos: ou é metilada pela TPMT para 6-metilmonofosfato (6-MeTIMP); ou é convertida pela inosina-5-monofosfato desidrogenase (IMPDH) em 6-tioxantosina monofosfato (6-TXMP), que, finalmente, pela ação da guanidina 5-monofosfato sintetase (GMPS), gera a 6-TGN e seus nucleotídeos ativos de efeito mielossupressor ${ }^{(24)}$.

Possivelmente outros produtos dessa reação, como o metilnitroimidazol, inibem o crescimento celular e levam à morte em conseqüência de danos em macromoléculas, como ácidos nucléicos, proteínas e membranas ${ }^{(36)}$.

O mecanismo de citotoxicidade e a atividade imunossupressora da AZA se dão mediante rompimento dos ácidos nucléicos. Os nucleotídeos de tioguanina (6-TGN) produzidos são incorporados ao DNA como falsos metabólitos, interferindo na síntese protéica e impedindo o crescimento e a proliferação dos linfócitos $T$ e $B^{(1)}$.

Recentemente, Tiede et al. ${ }^{(38)}$ relataram outra possível explicação para os efeitos imunossupressores em linfócitos T. O metabólito ativo da 6-MP - a 6-TGTP - inibe a ação da proteína guanosina trifosfato, a Rac1, envolvida nos sinais de ativação da via da CD28 (Figura 1). As patologias auto-imunes e rejeições de transplantes são mediadas por essa proteína transmembrana ${ }^{(31)}$. A inibição da Rac1 impossibilita a ativação de genes importantes, alvos de cascatas, como a do gene antiapoptótico $B c l-x l$ e do fator nuclear $\kappa B$ (NF-kB). O interesse no estudo desse fator de transcrição decorre da quantidade de genes e processos celulares que o NF- $\kappa$ B regula, como desenvolvimento, plasticidade, morte e defesa celular ${ }^{(5,28)}$.

\section{Polimorfismo genético da TPMT}

A TPMT é uma enzima citoplasmática responsável pela metilação das tiopurinas. Não se conhece nenhum substrato natural para essa enzima, nem a sua real função biológica. A atividade enzimática da TPMT apresenta grande variabilidade entre indivíduos. O polimorfismo dessa enzima foi investigado por diversos pesquisadores $(1,16,20)$. Tem-se conhecimento de dois alelos selvagens e 16 mutantes para o gene que codifica a TPMT. Esses genes são encontrados com variada freqüência nas diferentes etnias. Os caucasianos apresentam freqüência trimodal de distribuição dos fenótipos da TPMT. Cerca de 0,3\% dos caucasianos são homozigotos para o alelo não-funcional, sendo a atividade de S-metilação da 6-MP ausente ou insignificante. De 6\% a $11 \%$ são heterozigotos e têm atividade enzimática inter-
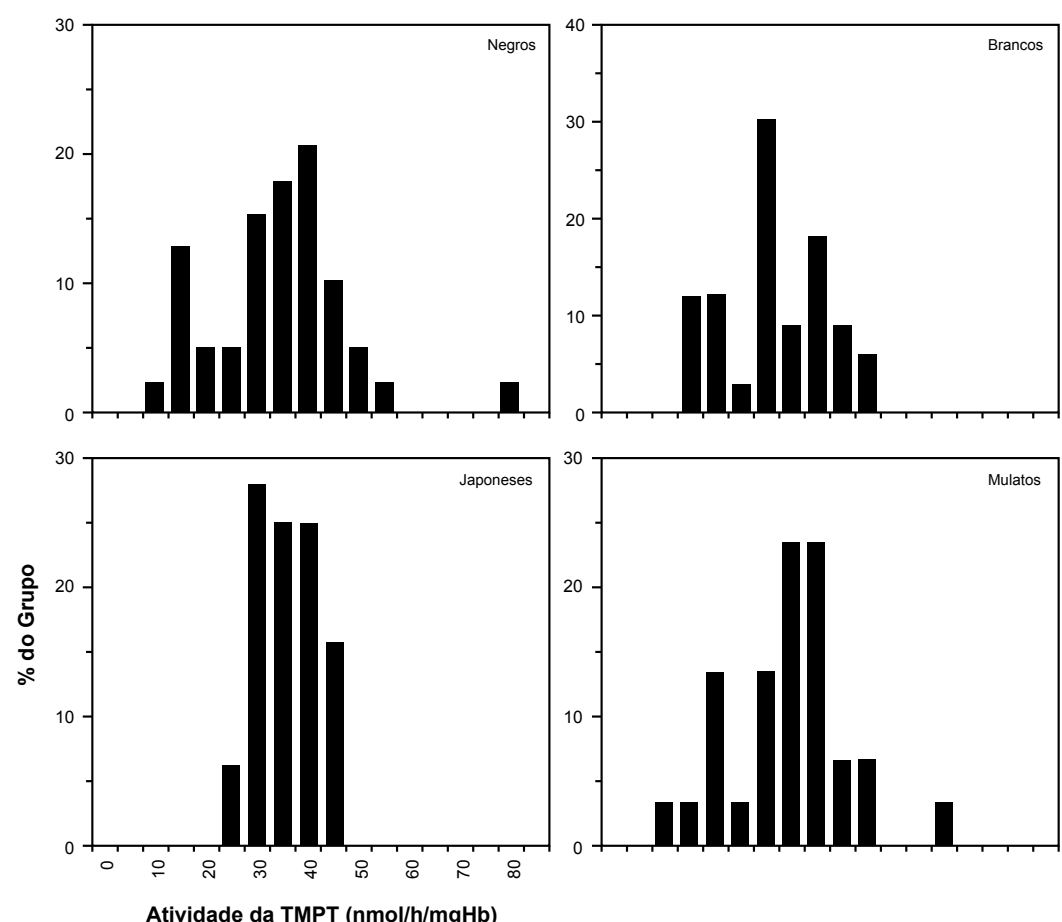

Atividade da TMPT (nmol/h/mgHb)

Figura 2 - Distribuição da atividade da TPMT nas diferentes populações de brasileiros. Adaptado de Chocair(10). 
mediária, enquanto $89 \%$ a $94 \%$ são homozigotos para o alelo selvagem e, conseqüentemente, apresentam níveis de atividade enzimática entre normal e alta.

A distribuição fenotípica da TPMT também foi pesquisada entre brasileiros. Nessa pesquisa observou-se que a atividade enzimática apresentou distribuição bimodal nas populações de brancos, negros e mulatos.

A população de japoneses não apresentou distribuição polimórfica, indicando que são homozigotos para algum alelo de metilação rápida ${ }^{(10)}$.

\section{Efeitos tóxicos}

O efeito tóxico mais comum da AZA é a depressão da medula óssea. Esse é um efeito dose-dependente que pode ser monitorado, resultando na redução da dose ou suspensão do medicamento. A contagem dos glóbulos vermelhos, brancos e plaquetas são recomendáveis a fim de monitorar esse efeito tóxico. A leucopenia é a manifestação hematológica mais freqüente, mas concentrações de 6 -TGN $>450 \mathrm{pmol} / 8 \times 10^{8}$ eritrócitos foram associadas à mielotoxicidade ${ }^{(17)}$.

Outro órgão alvo de efeitos tóxicos é o fígado. O mecanismo tóxico da AZA nos hepatócitos está associado ao primeiro passo da biotransformação, na qual há depleção dos níveis do detoxificador glutationa e aumento dos níveis de 6-MMP(14). Pacientes com níveis de 6-MMP $>5.700$ $\mathrm{pmol} / 8 \times 10^{8}$ eritrócitos apresentaram sinais de hepatotoxicidade ${ }^{(17)}$. A explicação pode estar na fase conjugação da AZA. Variações nos níveis de glutationa reduzida (GSH) afetam diretamente a síntese de proteínas e de DNA. A GSH encontrada nos hepatócitos é consumida, predispondo ao estresse oxidativo, com peroxidação lipídica e/ou lesão direta nas proteínas e nos ácidos nucléicos.

Lee e Farrell(23), utilizando culturas de hepatócitos de ratos, confirmaram que a AZA também causa morte celular dose-dependente, em seguida à depleção dos níveis de GSH. Nesse experimento, a adição de GSH ou $\mathrm{N}$-acetilcisteína preveniu contra morte celular. Determinar os níveis plasmáticos de enzimas hepáticas, como aspartato aminotransferase (AST) e alanina aminotransferase (ALT), é de grande valia para o acompanhamento de lesão celular hepática. A medida da atividade da fosfatase alcalina (ALP) e da bilirrubina total é um bom marcador de lesão tubular e da função hepática, respectivamente ${ }^{(27)}$.

Também se observou que alguns pacientes não respondem à terapia, mas eles apresentam os efeitos tóxicos devido a maior formação do metabólito 6-MMP. Chocair et al. ${ }^{(9)}$ sugerem que o alopurinol pode ser adicionado ao protocolo convencional desses pacientes, inibindo a XO que normalmente está em alta atividade sérica nas inflamações. Sparrow et al. ${ }^{(37)}$ demonstraram que a adição desse medicamento à terapia de pacientes que não respondem a AZA é um excelente modo de favorecer a formação dos nucleotídeos de 6-TCN, sem aumento dos níveis de 6-MMP. Cummins et al. (13) defendem que quando se decide usar a terapia combinada, a dose de AZA deve ser reduzida em até dois terços, tornando-se uma promissora opção na terapia imunossupressora.

\section{Monitoracão terapêutica}

Com o desenvolvimento de técnicas laboratoriais mais sensíveis e específicas, tornou-se possível a dosagem dos medicamentos imunossupressores nos fluidos biológicos. Algumas vezes é possível determinar a concentração de um fármaco em seu sítio de ação, como o metabólito 6-TGN, reduzindo-se assim as influências das variações farmacocinéticas.

A monitoração terapêutica de medicamentos não é indicada para todos os fármacos. Segundo Johnston e Holt(22), para que seja necessária a monitoração terapêutica de um fármaco, esse deve preencher os seguintes critérios:

- apresentar correlação entre concentração do fármaco e efeito terapêutico;

- estreita faixa terapêutica, isto é, pequenas variações nas doses podem causar reações adversas graves;

- grandes diferenças metabólicas entre indivíduos;

- sucesso terapêutico difícil de ser distinguido das reações adversas.

A importância da monitoração dos níveis de 6-TGNs intra-eritrocitários, com objetivo de reduzir a toxicidade dose-dependente, foi avaliada por diversos pesquisadores, demonstrando que a quantificação dos metabólitos da AZA exerce um papel importante no acompanhamento clínico de pacientes submetidos à terapia com $\operatorname{AZA}^{(11,12,17)}$.

Pacientes com deficiência de TPMT tratados com doses usuais de AZA apresentaram níveis altos de 6-TGN. As variações enzimáticas individuais da TPMT determinam a concentração dos metabólitos, e esses determinam a eficácia e os efeitos adversos. Estudos têm demonstrado uma relação de reciprocidade entre a atividade eritrocitária da TPMT e os níveis de 6-TGN eritrocitários, reforçando que a monitoração dos níveis de 6-MMP e 6-TGN intra-eritrocitários pode ser usada para individualizar a terapia. 
Banerjee e Bishop ${ }^{(4)}$ analisaram 101 pacientes pediátricos com DIls, recebendo doses de AZA, estáveis há, pelo menos, quatro meses. O grupo teste $(n=64)$ era formado por pacientes que tinham os metabólitos da AZA monitorados, enquanto no grupo controle $(n=37)$ os pacientes recebiam AZA sem a respectiva monitoração terapêutica, pois já faziam uso do medicamento anteriormente à implantação do método para dosagem da AZA no hospital. A freqüência de episódios de exacerbação da doença por paciente/ano foi $55 \%$ menor no grupo monitorado do que no grupo controle. O grupo teste também recebeu uma dose diária de prednisona $73 \%$ menor, em comparação com o grupo controle, no qual não se realizou a monitoração. Casos de mielotoxicidade foram encontrados em três dos 64 pacientes do grupo teste versus freqüência zero no grupo controle $(n=37)$. No entanto, todos os casos de leucopenia foram autolimitantes, mas não foi necessário reduzir a dose em nenhum dos pacientes. Observou-se elevação de enzimas hepáticas em seis dos $64(9,4 \%)$ pacientes do grupo teste, em oposição a seis de $37(16,2 \%)$ do grupo controle.

Bergan et al. ${ }^{(6)}$ relataram um estudo em dois grupos de transplantados renais, em que um grupo recebia doses baixas de AZA, $3 \mathrm{mg}$ no primeiro dia, $2 \mathrm{mg}$ na primeira semana e posterior manutenção com $1 \mathrm{mg}$; o segundo grupo recebia altas doses de AZA, 5 mg no primeiro dia, e ajuste das doses seguintes, visando nível de 6-TGN entre 100 e 200 pmol/8 $\times 10^{8}$ eritrócitos. A incidência acumulada de episódios de rejeição primária foi reduzida em $21 \%$ no grupo monitorado, mantendo-se a mielotoxicidade em níveis aceitáveis.

Dubinsky et al. ${ }^{(17)}$ avaliaram a utilidade clínica da monitoração da 6-TGN e da genotipagem da TPMT de pacientes pediátricos com Dll em tratamento com 6-MP ou AZA. Os autores demonstraram que os níveis de 6-TGN estavam associados à resposta terapêutica, e sugerem que a resposta a 6-MP/AZA poderia ser otimizada, com níveis de 6-TGN por volta de $235 \mathrm{pmol} / 8 \times 10^{8}$ eritrócitos.
Nos pacientes heterozigotos para um alelo mutante da TPMT, os níveis de 6-TGN eritrocitários foram significativamente maiores que nos heterozigotos que apresentavam o gene selvagem (média 589 versus $256 \mathrm{pmol} / 8 \times 10^{8}$ eritrócitos) ${ }^{(16)}$.

Alguns métodos já foram descritos para dosagem da 6-TGN e também para 6-MMP(7, 15, 19, 25, 29, 30). Essas metodologias apresentam algumas variações entre si, como na composição dos reagentes e nas concentrações preconizadas, mas, em geral, envolvem uma hidrólise ácida, com aquecimento em banho-maria seco, para conversão dos nucleotídeos de 6-TGN e 6-MMP em suas respectivas bases livres. Após filtragem, a amostra segue para uma fase de extração e separação em cromatografia liquida de alta eficiência (CLAE). As concentrações obtidas são expressas em pmol/8 x $10^{8}$ eritrócitos.

Até o momento não foram realizados estudos comparativos satisfatórios entre esses métodos, principalmente no que se refere à padronização dos procedimentos analíticos e expressão dos resultados.

\section{Conclusão}

A monitoração terapêutica proporciona uma série de benefícios para os pacientes que necessitam de terapias imunossupressoras. A resposta terapêutica - dependente das características genéticas do paciente - resulta em maior adesão ao tratamento devido à diminuição dos efeitos tóxicos.

Estudos retrospectivos indicam forte associação entre os níveis intra-eritrocitários de 6-TGN e o sucesso terapêutico, bem como altos níveis da 6-MMP para hepatotoxicidade e não-responsividade ao tratamento. Novos estudos poderão determinar o real benefício clínico da monitoração terapêutica precoce e periódica dos metabólitos da AZA.

\section{Referências}

1. AL HADITHY, A. F. Y. et al. Thiopurines in inflammatory bowel disease: pharmacogenetics, therapeutic drug monitoring and clinical recommendations. Dig Liv Dis, v. 37, p. 282-97, 2005

2. ARDIZZONE, S. Drugs used in ulcerative colitis. Orphanet, Paris: 2003. Disponível em: <http://www.orpha.net/ data/patho/GB/UC-drugs.pdf>. Acesso em: 10 set. 2007.

3. ARMSTRONG, V. W.; OELLERICH, M. New developments in the immunosuppressive drug monitoring of cyclosporine, tacrolimus, and azathioprine. Clin Biochem, v. 34, p. 9-16, 2001.

4. BANERJEE, S.; BISHOP, W. P. Evolution of thiopurine use in pediatric inflammatory bowel disease in an academic center: role of thiopurine methyltransferase and 6-mercaptopurine metabolite measurements. Gastroentereology, v. 43, n. 3, p. 324-30, 2006.

5. BARNES, P. J.; KARIN, M. Nuclear factor- $\mathrm{KB}$ : a pivotal 
transcription factor in chronic inflammatory diseases. N Engl J Med, v. 336, p. 1066-71, 1997.

6. BERGAN, S. et al. Monitored high-dose azathioprine treatment reduces acute rejection episodes after renal transplantation. Transplantation, v. 66, n. 3, p. 334-9, 1998.

7. BOULIEU, R. etal. High-performance liquid chromatographic determination of thiopurine metabolites of azathioprine in biological fluids. J Chromatogr, v. 615, p. 352-6, 1993.

8. BRASÍLIA. Ministério da Saúde. Portal da Saúde. Doença de Crohn. Brasília, 2002. Disponível em: <http://portal. saude.gov.br/saude/visualizar_texto.cfm?idtxt=23529>. Acesso em: 8 mar. 2007.

9. CHOCAIR, P. R. et al. Low-dose allopurinol plus azathioprine/ cyclosporin/prednisolone, a novel immunosuppressive regimen. Lancet, v. 342, p. 83-4, 1993.

10. CHOCAIR, P. R. Contribuição para o estudo da azatioprina: potencialização de sua atividade imunossupressora pelo alopurinol em pacientes com transplante renal. São Paulo, 1994. Tese (Livre-docência) - Faculdade de Medicina da Universidade de São Paulo.

11. CUFFARI, C. et al. 6-Mercaptopurine metabolism in Crohn's disease: correlation with efficacy and toxicity. Gut, v. 39, p. 401-6, 1996.

12. CUFFARI, C. et al. Utilization of erythrocyte 6-thioguanine metabolite levels to optimize azathioprine therapy in patients with inflammatory bowel disease. Gut, v. 48, p. 642-6, 2001.

13. CUMMINS, D. et al. Myelosuppression associated with azathioprine allopurinol interaction after heart and lung transplantation. Transplantation, v. 61, n. 11, p. 1661-2, 1996.

14. DELEVE, L. D. etal. Toxicity of azathioprine and monocrotaline in murine sinusoidal endothelial cells and hepatocytes: the role of glutathione and relevance to hepaticvenoocclusive disease. Hepatology, v. 23, p. 589-99, 1996.

15. DERVIEUX, T.; BOULIEU R. Simultaneous determination of 6-thioguanine and methyl 6-mercaptopurine nucleotides of azathioprine in red blood cells by HPLC. Clin Chem, v. 44, p. 551-5, 1998.

16. DUBINSKY, M. C. et al. Pharmacogenomics and metabolite measurement for 6-mercaptopurine therapy in inflammatory bowel disease. Gastroenterology, v. 118, p. 705-13, 2000.

17. DUBINSKY, M. C. et al. Optimizing immunomodulator therapy for inflammatory bowel disease. Curr Gastroenterol Rep, v. 5, p. 506-11, 2003.

18. EKLUND, B. I. Role of multiple g/utathione transferases in bioactivation of thiopurine prodrugs. Studies of Human Soluble Glutathione Transferases from Alpha, Kappa, Mu, Omega, Pi, Theta, and Zeta Classes. Uppsala, 2006. Thesis (Doctoral) - Faculty of Science and Technology Acca Universitet Upsaliensis.

19. ERDMANN, G. R. etal. Reversed-phase high-performance liquid chromatographic approach to determine total lymphocyte concentrations of 6-thioguanine, methylmercaptopurine and methylthioguanine in humans. J Chromatogr, v. 571, p. 149-56, 1991.
20. GEARRY, R. B.; BARCLAY, M. L. Azathioprine and 6mercaptopurine pharmacogenetics and metabolite monitoring in inflammatory bowel disease. $J$ Gastroent Hepatol, v. 20, n. 8, p. 1149, 2005.

21. GOODMAN, L. S.; GILMAN, A. The pharmacological basis of therapeutics. São Paulo, SP: Mcgraw-Hill, 2003.

22. JOHNSTON, A.; HOLT, D. W. Therapeutic monitoring of immunosuppressant drugs. Br J Clin Pharmacol, v. 47, p.339-50, 1999.

23. LEE, A. U.; FARRELL, G. C. Mechanism of azathioprine induced injury to hepatocytes: roles of glutathione depletion and mitochondrial injury. J Hepatol, v. 35, n. 6, p. 756-64, 2001.

24. LENNARD, L. The clinical pharmacology of 6-mercaptopurine. Eur J Clin Pharmacol, v. 43, p. 32-9, 1992.

25. LENNARD, L.; SINGLETON, H. J. High-performance liquid chromatographic assay of human red blood cell thiopurine methyltransferase activity. J Chromatogr $B$ Biomed Sci Appl, v. 661, p. 25-33, 1994.

26. MCLEOD, H. L. Therapeutic drug monitoring opportunities in cancer therapy. Pharmacol Ther, v. 74, n. 1, p. 3954, 1997.

27. NAVARRO, V. J.; SENIOR, J. R. Drug-related hepatotoxicity. N Engl J, v. 354, p. 731-9, 2006.

28. O’NEILL, L. A. J.; KALTSCHMIDT, C. NF-kB: a crucial transcription factor for glial and neuronal cell function. Trends Neuroscience, v. 20, p. 252-8, 1997.

29. OLIVEIRA, B. M. et al. An improved HPLC method for the quantitation of 6-mercaptopurine and its metabolites in red blood cells. Braz J of Med Biol Res, v. 37, p. 649-58, 2004.

30. PIKE, M. G. et al. Improved methods for determining the concentration of 6-thioguanine nucleotides and 6-methylmercaptopurine nucleotides in blood. $J$ Chromatogr B, v. 757, p. 1-9, 2001.

31. RATHMELL, J. C.; THOMPSON, C. B. Pathways of apoptosis in lymphocyte development, homeostasis, and disease. Cell, v. 109, n. 2, Supplement 1, p. 97-107, 2002.

32. SAMBROOK, P. N. Comparison of methotrexate with azathioprine or 6-mercaptopurine in refractory rheumatoid arthritis: a life table analysis. $\mathrm{Br} \mathrm{J}$ Rheumatol, v. 25, p. 372-5, 1986.

33. SANDBORN, W. J. et al. Lack of effect of intravenous administration on time to respond to azathioprine for steroid-treated Crohn's disease. Gastroenterology, v. 117, p. 527-35, 1999.

34. SANDBORN, W. J. et al. Azathioprine or 6-mercaptopurine for inducing remission of Crohn's disease (Cochrane review). In: The Cochrane Library, Issue 1. Oxford: Update Software, 1999.

35. SÃO PAULO (SP). Associação Brasileira de Transplante de Órgãos. Estatísticas em transplantes. São Paulo, 2007. Disponível em: <http://www.abto.org.br/profissionais/ profissionais.asp>. Acesso em: 1 set. 2007.

36. SAUER, H.; HANTKE, U.; WILMANNS, W. Azathioprine lymphocytotoxicity: potentially lethal damage by its imidazole derivatives. Arzneimittelforschung, v. 38, p. 820-4, 1988.

37. SPARROW, M. P. et al. Allopurinol safely and effectively 
optimizes thioguanine metabolites in inflammatory bowel disease patients no responding to azathioprine and mercaptopurine. Aliment Pharmacol Ther, v. 22, p. 441-6, 2005.
38. TIEDE, I. et al. CD28-dependent Rac1 activation is the molecular target of azathioprine in primary human CD4+ T Iymphocytes. J Clin Invest, v. 111, p. 113345, 2003.

Maurílio Pacheco Neto

Setor de Toxicologia - Serviço de Bioquímica Clínica

Divisão de Laboratório Central do HC/FMUSP

Av. Dr. Enéas de Carvalho Aguiar, $155 / 2^{\circ}$ andar (Bloco 8) - Cerqueira César

CEP: 05403-010 - São Paulo-SP

Tel.: (011) 3069-6109

Fax: (011) 3083-2498

e-mail: mauriliopacheco@hotmail.com 